\title{
Efficiency of Propane-Air Mixture Combustion Assisted by Deeply Undercritical MW Discharge in Cold High-Speed Airflow
}

\author{
Igor I. Esakov, ${ }^{\dagger}$ Lev P. Grachev, ${ }^{\ddagger}$ Kirill V. Khodataev, ${ }^{\S}$ \\ Moscow Radiotechnical Institute RAS, Moscow, 117519, Russia, \\ Viacheslav A. Vinogradov ${ }^{* *}$ \\ Central Institute of Aviation Motors, Moscow, 111116, Russia, \\ and \\ David M. Van Wie $\mathrm{Wi}^{\dagger \dagger}$ \\ The John Hopkins University Applied Physics Laboratory, Laurel, MD, USA
}

\begin{abstract}
Results are presented from an experimental investigation of propane-air combustion in a stationary microwave discharge at different conditions. In these experiments, a deeply undercritical discharge is initiated in the base of an electromagnetic vibrator, which is immersed in cold supersonic airflow. Two schemes for mixing propane with air are considered. In the first scheme, a propane-air mixture is delivered through the vibrator and is ignited at the outlet from the vibrator. In the second scheme, propane delivered through the vibrator mixes with air captured by an inlet hole in the vibrator nose prior to being also ignited by the MW discharge at the base of the vibrator. Propane combustion with efficiency of approximately $60 \%$ was demonstrated at a mixture velocity up to $200 \mathrm{~m} / \mathrm{s}$ in the combustion region. The heat release reaches approximately $1 \mathrm{~kW}$ at a propane mass flow rate of $2 \cdot 10^{-2} \mathrm{~g} / \mathrm{s}$ and discharge power of approximately $200 \mathrm{~W}$.
\end{abstract}

\section{Introduction}

$\mathbf{R}$ esults from several successive stages of experimental investigations are presented in this article. These experiments have been devoted to the study the ignition and combustion characteristics of gaseous propaneair mixtures in a supersonic (SS) air stream in the region of a microwave (MW) gaseous discharge. Results from earlier stages of these investigations were presented in references [1] and [2].

In these experiments we create a discharge in the linear polarized quasi-optical beam of electromagnetic (EM) radiation. The discharge is initiated in the EM field through the use of a vibrator at a level that is dozens of times smaller than the critical one. Recall that the critical breakdown field is the minimum field necessary for electrodeless gas breakdown at its pressure $\boldsymbol{p}$. The vibrator used for this purpose is a linear electromagnetic antenna and is applied with its axis collinear to the direction of the SS stream velocity and to the electric component $\boldsymbol{E}_{\boldsymbol{0}}$ of the initial EM field. The discharge in this case is realized at the base of the initiating vibrator in a quasi-steady mode during whole time $\tau_{M W}$ that the EM radiation exists. The discharge plasma is in contact with the base of the vibrator during entire time of the discharge $\tau_{d i s}=\tau_{M W}$. The area of the discharge itself elongates along the stream.

*Copyright (C) 2006 by the American Institute of Aeronautics and Astronautics, Inc. All rights reserved

${ }^{\dagger}$ Principal Investigator.

* Principal Investigator.

$\S$ Professor, Head of Plasma Physics department, member AIAA

${ }^{* *}$ Principal Investigator, Associate Fellow AIAA

${ }^{\dagger}$ Principal Professional Staff, Associate Fellow AIAA . 
During the experiments, pure gaseous propane $\mathrm{C}_{3} \mathrm{H}_{8}$ or its mixture with air is injected into the discharge area during time $\boldsymbol{\tau}_{i n j}$. The propane mass flow rate $\boldsymbol{m}_{p r}$ and air-fuel ratio $\boldsymbol{r}=\boldsymbol{m}_{a i r} / \boldsymbol{m}_{p r}$ (where $\boldsymbol{m}_{a i r}$ is air mass flow rate) are varied for different experiments.

Experiments were conducted to determine the conditions for generating the energy release region, the geometric characteristics of the discharge and the color distribution of its emission. Spatial distributions of stagnation pressure $\boldsymbol{p}_{\text {stag }}$ and stagnation temperature $\boldsymbol{T}_{\text {stag }}$ are measured in the wake of the energy release region. Analysis of these data allows one to estimate quantitatively the amount of EM radiation energy that was put into air heating in the discharge plasma and the percentage of propane combustion.

\section{Investigation results during initial stages}

Let us outline the main results of the previous stage of our investigations that were presented in References [1] and [2]. In these experiments we initiated a deeply undercritical MW discharge as indicated above. A quasi-optical EM beam of power $\boldsymbol{P}_{\boldsymbol{M W}}$ was used to create the discharge, which was observed at incident power levels a thousand times smaller than the power required to ensure electrodeless air breakdown.

Initially, it was unclear if this type of low-energy discharge could be used to ignite a propane-air flammable mixture, even at slow speeds. Our specially executed experiment demonstrated this possibility which can be seen in Fig.1. The discharge itself is shown in the left photo where it is observed that the discharge burns at the ends of the vertical vibrator that was used to initiate the discharge. In the right photo one can see the discharge and the combustion of a propane-air mixture which is delivered from below through the tube visible at the bottom of the photo.
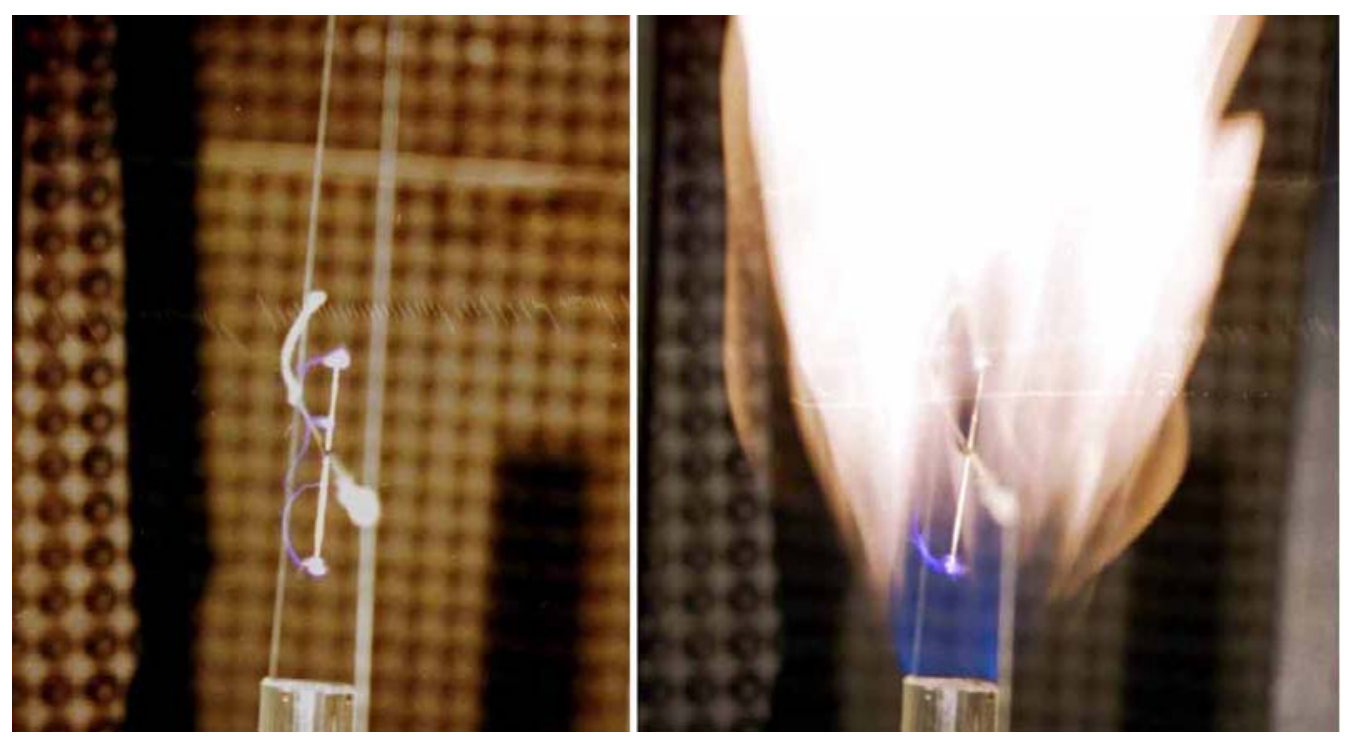

Figure 1. The deeply undercritical discharge, initiated by the vibrator with $2 a=0.25 \mathrm{~cm}$ and $2 L=4 \mathrm{~cm}\left(E_{0}=1.5 \mathrm{kV} / \mathrm{cm}\right.$,): left photo - without flow of the flammable mixture, right photo - with the flow of the flammable mixture.

During the next series of experiments, the vibrator initiating the discharge was located in an air stream, and propane was injected into the discharge region through a hole in the base of the vibrator. The air velocity $\boldsymbol{V}$ in the stream was gradually increased to SS speeds during successive experiments. In Fig.2 one can see the corresponding illustrative photos. The initiator is placed horizontally in these photos, and direction of the air stream is from left to right. The discharge area is attached to the vibrator base and a combusting propane torch of typical blue color can be seen in photos. The length of the propane combustion region (along the stream) decreases with increasing velocity $V$.

Measurements of $\boldsymbol{p}_{\text {stag }}$ and $\boldsymbol{T}_{\text {stag }}$ in the initiator wake demonstrated propane combustion in this experimental formulation up to a value $\boldsymbol{V}=500 \mathrm{~m} / \mathrm{s}$, which corresponds to Mach number $M=2$ at the experimental conditions. Sample temporal waveforms of $\boldsymbol{p}_{\text {stag }}$ and $\boldsymbol{T}_{\text {stag }}$ are presented in Fig.3a and Fig.3b, respectively. In this figure one can see two waveforms: one due to the MW discharge itself and one due to the combined effect of the MW discharge and the propane combustion. The $\boldsymbol{p}_{\text {stag }}$ in the stream, as measured using a Pitot tube, decreases when the discharge is initiated, and decreases even more under the combined effect of the discharge and propane combustion. In contrast, 
the discharge heats the gas and the temperature $\boldsymbol{T}_{\text {stag }}$ increases, and upon the introduction of the combusting propane, the temperature increases further.

In Fig.4 one can see typical $\boldsymbol{T}_{\text {stag }}$ distributions in the wake of the vibrator at $M=2$ in a plane perpendicular to the stream with only the discharge (see left illustration) and under the combined effect of the discharge and combustion (see right photo). Analysis of the $\boldsymbol{p}_{\text {stag }}$ and $\boldsymbol{T}_{\text {stag }}$ distributions for the case with the discharge only showed that the energy input at $M=2$ and $P_{\mathrm{MW}}=1.5 \mathrm{~kW}$ was accompanied by air heating in the discharge zone that reaches $P_{\text {dis }} \approx 150 \mathrm{~W}$, which is approximately $10 \%$ of $\boldsymbol{P}_{M W}$.

Using this scheme, the experiments provide a sense of the amount of propane combustion that can be realized using pure propane injection into the discharge. Under these conditions, there is an optimum propane mass flow rate that can be determined. By optimal mass flow rate $\boldsymbol{m}_{p r}$ we mean the amount producing the maximum energy release from propane-air combustion for a known rate of air mass flow. The measured power $\boldsymbol{P}_{\text {com }}$ released with propane combustion decreases with respect of the maximum value with decreasing $\boldsymbol{m}_{\boldsymbol{p} r}$. The power of the propane combustion is $\boldsymbol{P}_{\text {com }} \approx 280 \mathrm{~W}$ at an optimum $\boldsymbol{m}_{\mathrm{pr}}=8.6 \cdot 10^{-3} \mathrm{~g} / \mathrm{s}$. Finally, the experiments show that the released power does not increase further with increasing $\boldsymbol{m}_{p r}$. The power associated with complete propane combustion is $\boldsymbol{P}_{\text {com max }}=400 \mathrm{~W}$ for an optimum value of $\boldsymbol{m}_{p r}$, so the results indicate that about $70 \%$ of the propane is combusted at the optimum propane mass flow rate in this experiment.

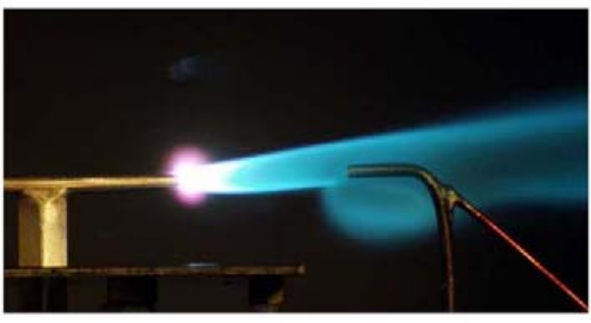

$3 \mathrm{~m} / \mathrm{s}$

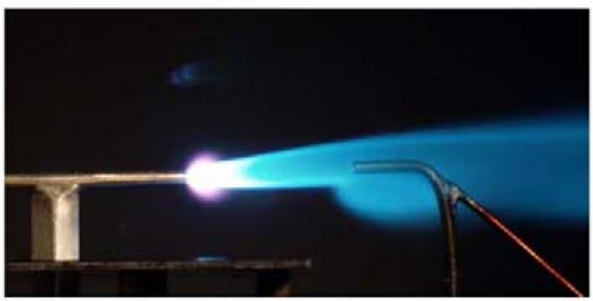

$12 \mathrm{~m} / \mathrm{s}$

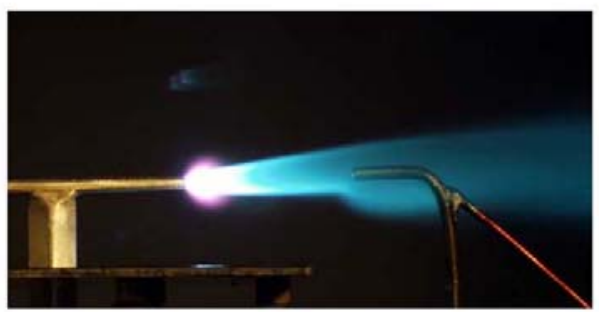

$30 \mathrm{~m} / \mathrm{s}$

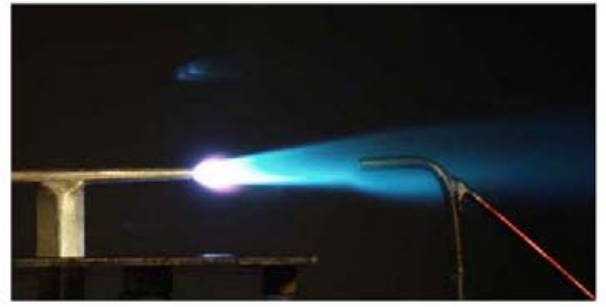

$50 \mathrm{~m} / \mathrm{s}$

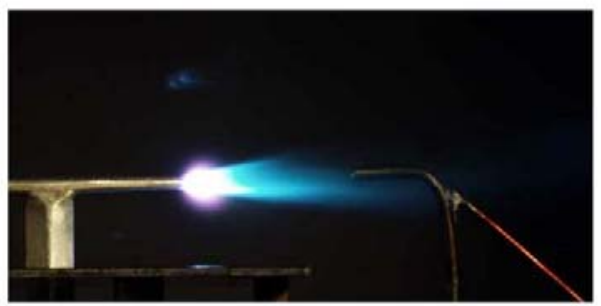

$85 \mathrm{~m} / \mathrm{s}$

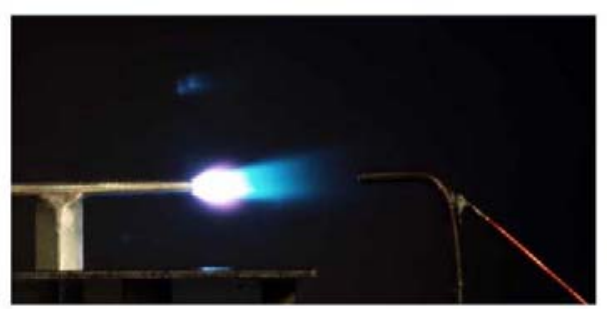

$200-500 \mathrm{~m} / \mathrm{s}$

Figure 2. The MW discharge in airflow at varying velocity with pure propane injection. 


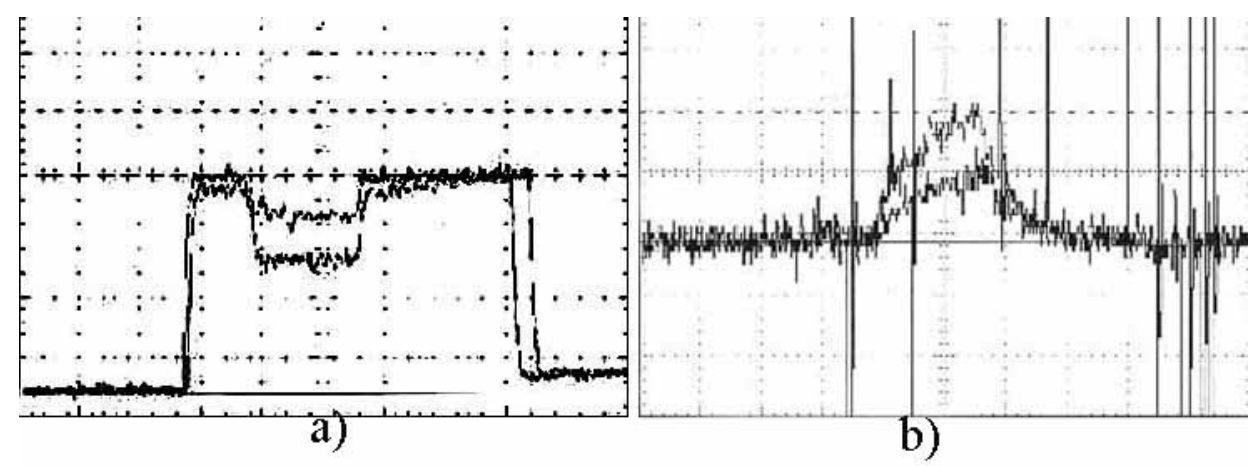

Figure 3. Waveforms of $p_{\text {stag }}$ (a) and $T_{\text {stag }}$ (b) due to the MW discharge only (upper one) and due to the impact of both the MW discharge and propane combustion (lower one).

In these experiments, the processes of propane-air mixing, ignition of the formed flammable mixture and its combustion in the base of the initiating vibrator were not controlled, so the burning process is very complicated to diagnose and control. This study was executed by monitoring of the parameters in the wake as a function of input power.
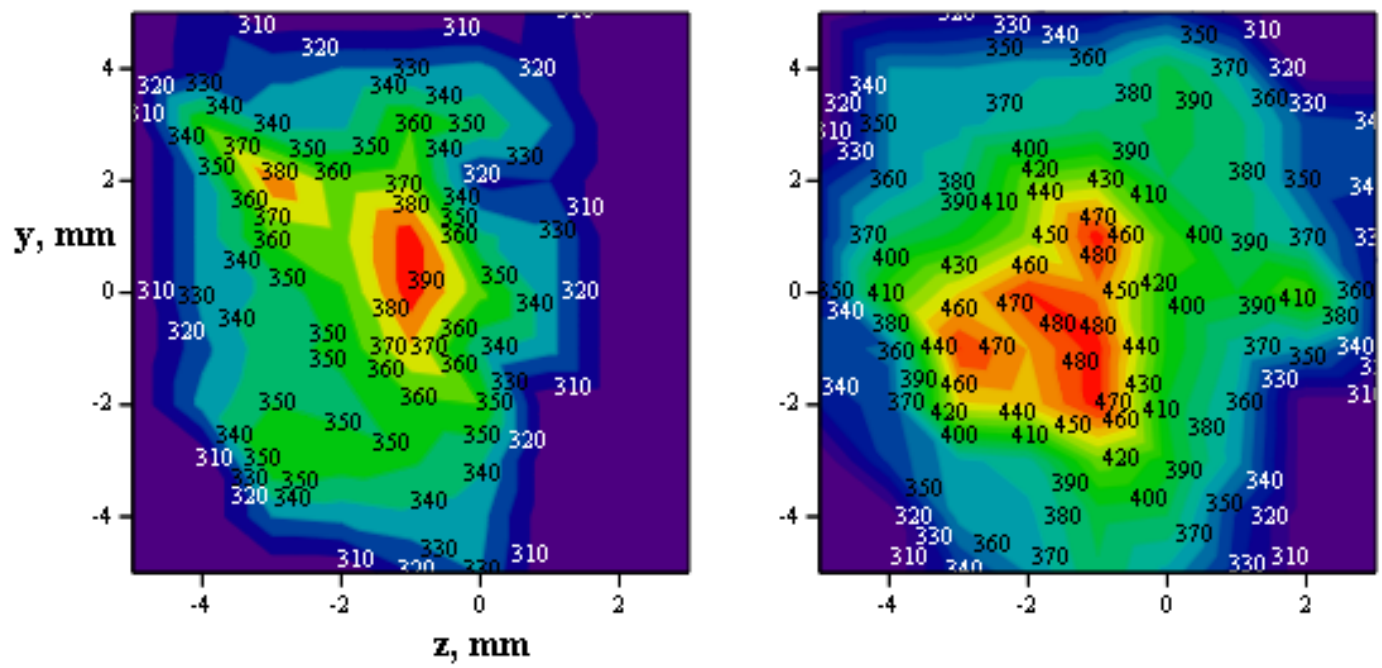

Figure 4. Typical $T_{\text {stag }}$ distributions in the vibrator's trail at $M=2$ in a plane perpendicular to the stream at burning of the discharge only (left) and at additional propane injection (right).

A goal of the experiments executed during the present stage was the development of a process control for which propane combustion efficiency can be maximized at a given energy input level in the discharge. This requires realization of effective propane-air mixing before reaching the discharge region where the ignition and combustion of the propane-air mixture occurs. To meet this requirement, air captured by an inlet device is ducted to an internal channel in the vibrator where it is mixed with propane. This mixture is injected in the base of the vibrator where ignition and combustion occurs.

\section{Experimental set up}

The experimental setup used in our experiments is described in detail in Reference [2]. In this paper, we briefly describe its main characteristics. A schematic of the setup is provided in Fig. 5a.

The test chamber is initially pumped down to the desired test pressure. Upon initiation of the experiment, air flows from the atmosphere to the test chamber through an axisymmetric SS Laval nozzle whose exit diameter is $30 \mathrm{~mm}$. A SS airstream is formed at the outlet of the nozzle with a Mach number $M=2$ and static pressure $p_{\mathrm{st}} \approx 100$ Torr, static temperature $T_{\mathrm{st}}=150 \mathrm{~K}$ and stream velocity $v \approx 500 \mathrm{~m} / \mathrm{s}$. The freestream stagnation conditions are $p_{\text {stag }} \approx 550$ Torr and $T_{\text {stag }}=300 \mathrm{~K}$. A submerged jet is formed in the test chamber at an air pressure equal to 
$p_{\mathrm{c}} \approx 100$ Torr and an $18 \mathrm{~mm}$ diameter core exists with uniform conditions over an axial distance of $12 \mathrm{~cm}$ from the nozzle exit.

In the experiments, a linear antenna vibrator is placed along the axis of the SS stream; its length is $\boldsymbol{2} \boldsymbol{L}$ and diameter is $2 \boldsymbol{a}$. The center cross section of the vibrator is placed at a distance of $6 \mathrm{~cm}$ from the outlet of the Laval nozzle. The vibrator is held by a streamlined pylon at a height $\boldsymbol{H}$ over a metallic screen, which is located outside the SS stream. A linearly polarized beam of EM radiation with a wavelength of $\lambda=12.5 \mathrm{~cm}$, power $P_{\mathrm{MW}}=1.5 \mathrm{~kW}$ and a typical transverse size $9 \mathrm{~cm}$ is introduced perpendicular to the screen surface. The amplitude of the initial EM field is $E_{0} \approx 200 \mathrm{~V} / \mathrm{cm}$ in the region of the initiator. For reference, the breakdown amplitude for the critical field is $E_{\mathrm{cr}}=8.4 \mathrm{x} \cdot 10^{3} \mathrm{~V} / \mathrm{cm}$ at $p_{\mathrm{st}}=100$ Torr and $T_{\mathrm{st}}=150 \mathrm{~K}$. So, the degree of undercriticality of the initial field is equal to 42.

Propane or a propane-air mixture (in any given proportion) can be injected during a time $\tau_{i n j}$ through internal tubes in the pylon and vibrator.

In Fig. $5 \mathrm{~b}$ the timeline of the operation is shown. One can see that SS stream is on for a time of $\tau_{\mathrm{fl}}=1.1 \mathrm{~s}$, the MW discharge during $\tau_{\text {dis }}=0.4 \mathrm{~s}$, and fuel is injected during $\tau_{\text {inj }}=0.5 \mathrm{~s}$. Calibration and control experiments were carried out with and without the airstream, with and without the discharge, and with and without the fuel injection.

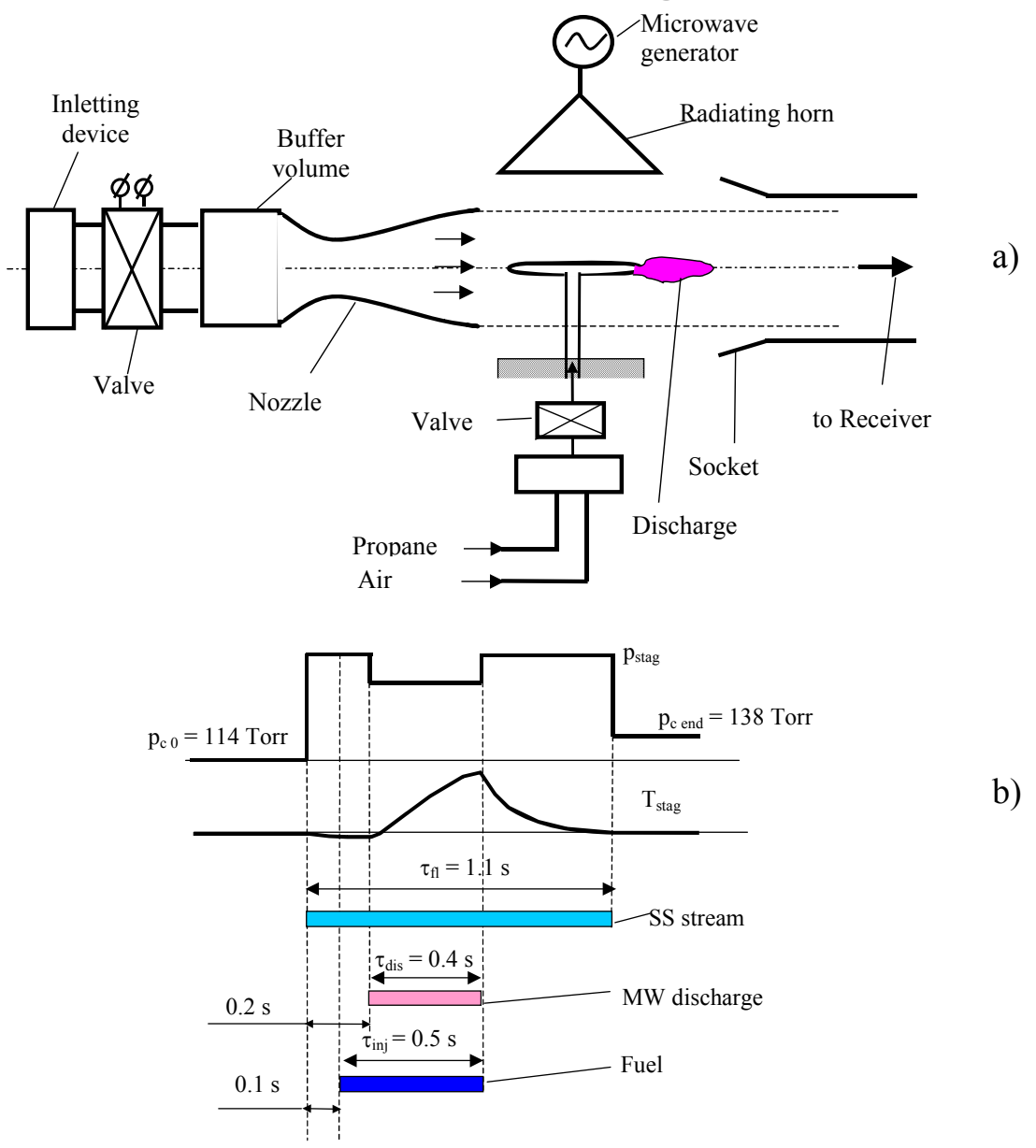

Figure 5. Set up scheme (a) and the timeline of operation of experimental setup (b).

\section{Experiments with an initiating vibrator}

Preliminary experiments were conducted to investigate the impact of a substantial enlargement of the vibrator diameter and the form of its aft end on its ability to initiate a MW discharge.

In Fig. 6 one can see data obtained in motionless air. In these experiments we changed the length $2 L \leq \lambda / 2$ of tube-type copper vibrators with flat ends of different diameters $2 a$, and we determined the maximum pressure $p_{\text {br }}$ in the test chamber for which air breakdown still occurs. A typical photo of the resulting MW discharge is also shown in Fig. 6. 


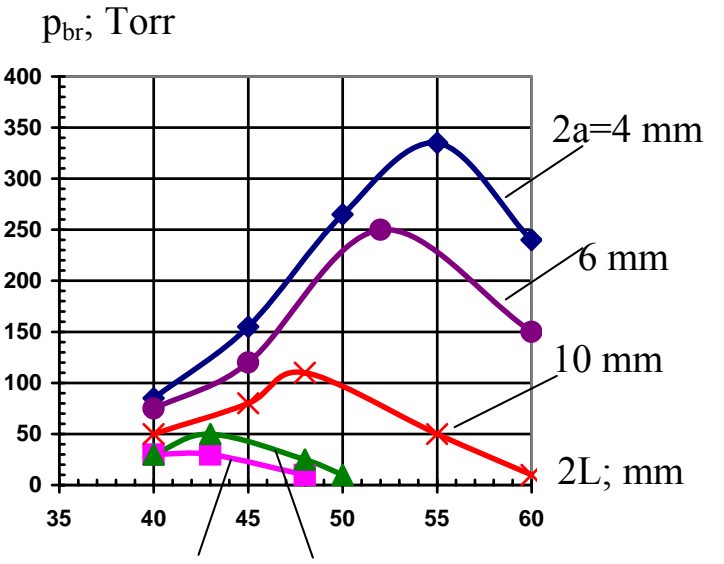

$18 \mathrm{~mm} \quad 12 \mathrm{~mm}$

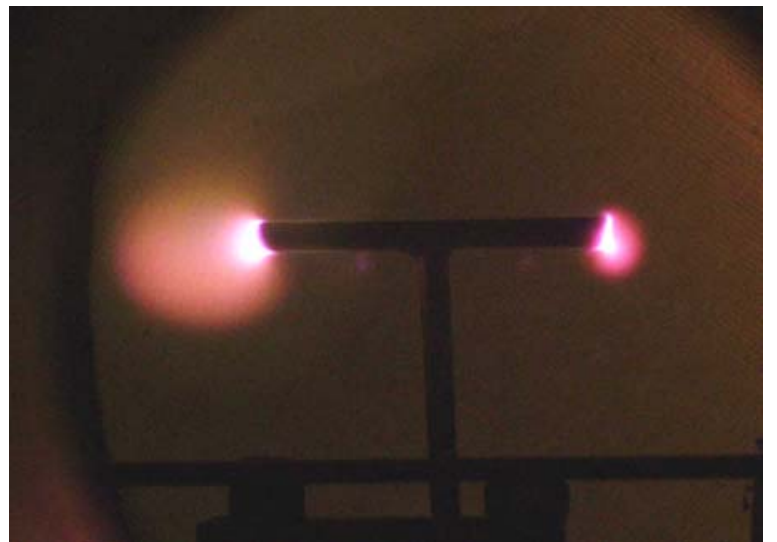

Figure 6. Experimental dependencies of maximum pressure in the working chamber, at which MW discharge was still realized (to the left) and appearance MW discharge in motionless air (to the right).

It follows from Fig. 6 that at each $2 a$, for $2 \mathrm{a}<18 \mathrm{~mm}$, there is a specific length $2 L$, at which breakdown is possible at a maximum $p_{b r}$. Experiments were then carried out with vibrators of this optimal length, for which air breakdown takes place most reliably. The optimal height $H$ was experimentally selected too, and found to be slightly smaller than $\lambda / 4$.

Experiments in a SS stream showed that the discharge cannot be initiated without a corresponding profiling of the tube-type vibrator base part for $2 a>6 \mathrm{~mm}$. The results of this experimental cycle are clear from the schematic presented in Fig. 7.

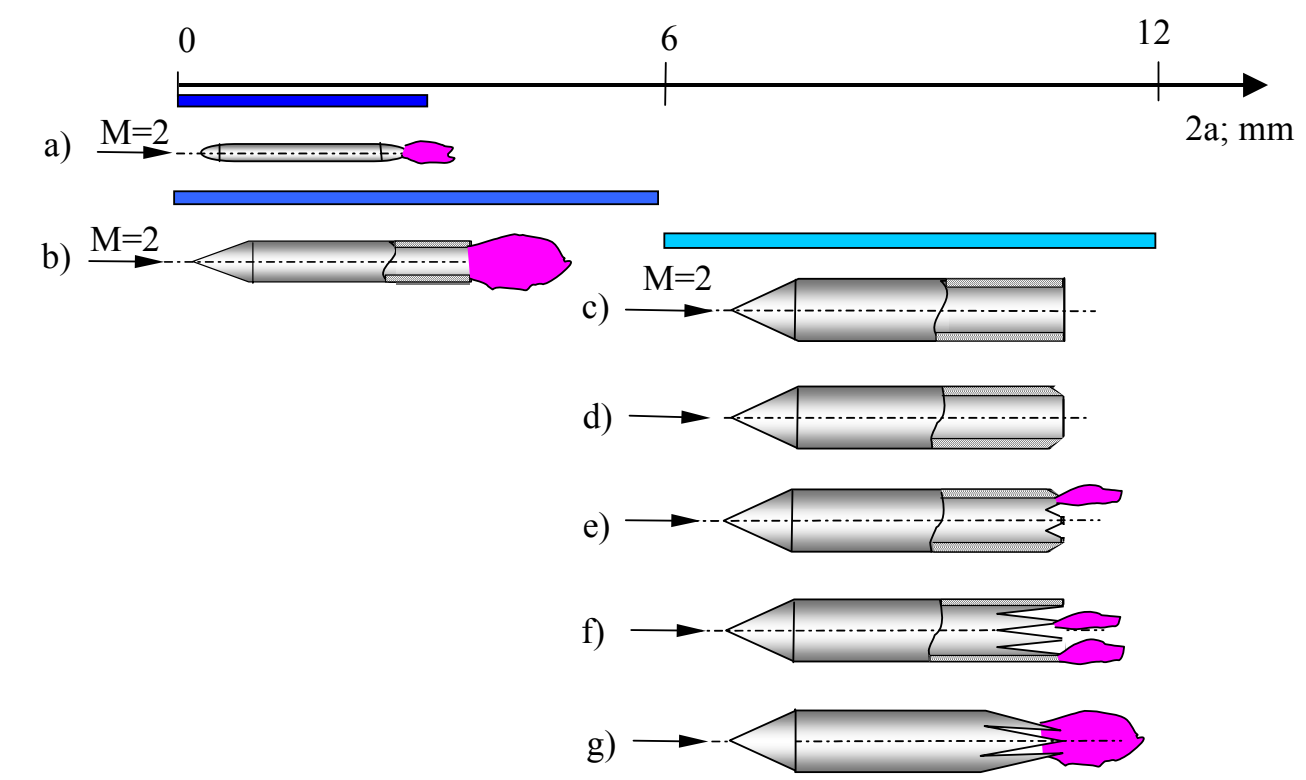

Figure 7. The scheme of search of the optimal form of initiating vibrator for mixture ignition

A tube-type vibrator with $2 a=12 \mathrm{~mm}, 2 L=42 \mathrm{~mm}$ and $H=24 \mathrm{~mm}$ was applied in the next series of experiments. For these experiments a dielectric fairing was placed on the nose of the vibrator, and its base was finished with 6 teeth of length $10 \mathrm{~mm}$ uniformly distributed around the tube perimeter. The ends of the sharpened teeth were drawn up to diameter of $2 c=5.5 \mathrm{~mm}$.

In Fig. 8 one can see photos of the MW discharge initiated by this vibrator in the SS stream. The exposure time was $\tau_{\mathrm{ex}}=1 \mathrm{~s}$ (in the left photo) and $\tau_{\mathrm{ex}}=10^{-3} \mathrm{~s}$ (in the right photo). Typical values of the maximum transverse size of plasma region in experiments did not exceed $12 \mathrm{~mm}$ while the length was about $19 \mathrm{~mm}$. 

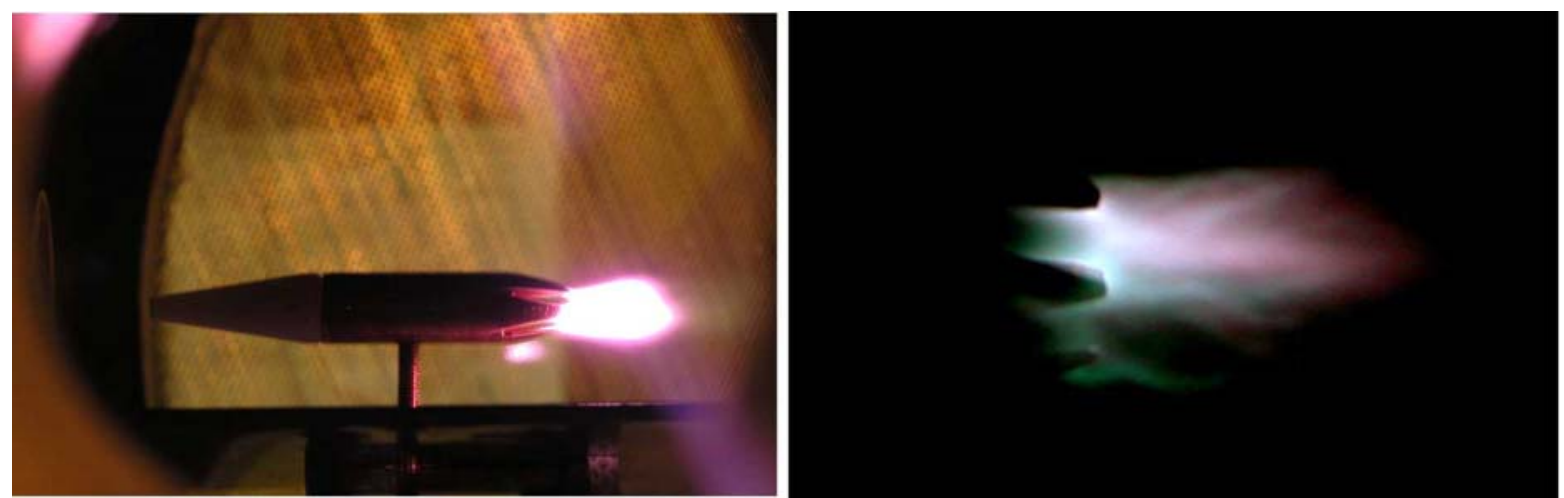

Figure 8. MW discharge initiated by optimal vibrator in SS stream, exposure time was $\tau_{\mathrm{ex}}=1 \mathrm{~s}$ (in the left) and $\tau_{\mathrm{ex}}=10^{-3} \mathrm{~s}$.

\section{Experiments with pre-mixed flammable mixture delivered through vibrator}

This experimental series was devoted to the development of the design for the vibrator base with the aim of ensuring effective propane combustion with the help of an initiated MW discharge. In Fig. 9a a schematic illustrates the scheme for injection of a pre-mixed propane-air mixture through an internal channel in the vibrator.

The experimental results were similar to those described earlier: the optimum amount of energy release occurs with injection of pure propane; the amount of propane combusted initially increases with increasing $\boldsymbol{m}_{\boldsymbol{p r}}$ prior to reaching a level where it practically does not change with further increases in the fuel flow; and finally the waveform amplitudes of $\boldsymbol{T}_{\text {stag }}$ are nearly identical to the values that were obtained in the initial experiments [2] and were presented in Fig. 3b.

This picture changed when the base of the vibrator was equipped with a dielectric tube as shown in Fig.9b. When tested in this configuration, an optimum air/fuel ratio, $\boldsymbol{r}$, was determined. In Fig.10 typical waveforms of $\boldsymbol{T}_{\text {stag }}$ are presented together with the corresponding photographs of the energy release region for a series of experiments conducted at a constant mixture delivery pressure. The corresponding value of $\boldsymbol{r}$ is given for each case.

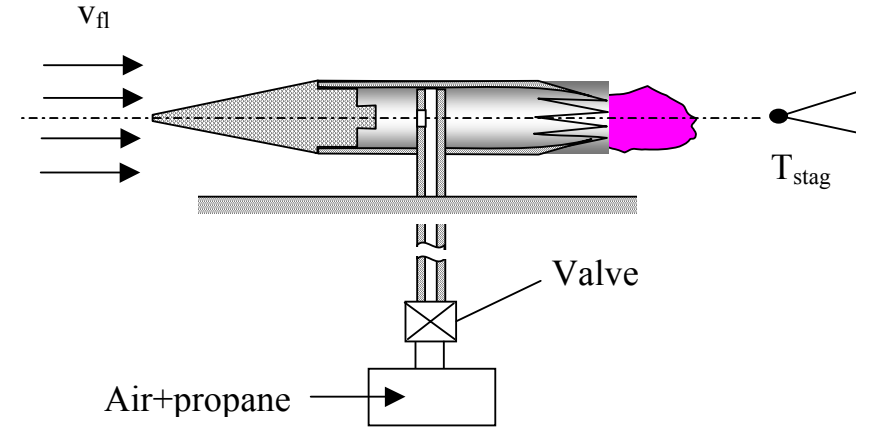

a)

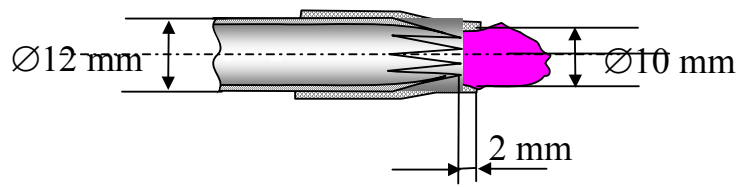

b)

Figure 9. Scheme of internal initiator channel design for injection of a pre-mixed propane-air mixture a) and scheme the base area a vibrator with a nozzle (b).

One can see in Fig.10 that the temperature amplitudes of the signal in the cases with the MW discharge in pure air and in pure propane are practically the same with a small temperature rise registered in both cases. The maximum temperature increase occurs in the range $\boldsymbol{r}=11-14$. Recall that the stoichiometric ratio is $\boldsymbol{r} \approx 15.7$. In Fig. 10 one can also see that the color of the energy release zone is blue in for the case with pure propane injection and pink for the case with pure air. The propane color gradually disappears with increasing air content in the mixture and is practically absent at the optimum value of $\boldsymbol{r}$. 

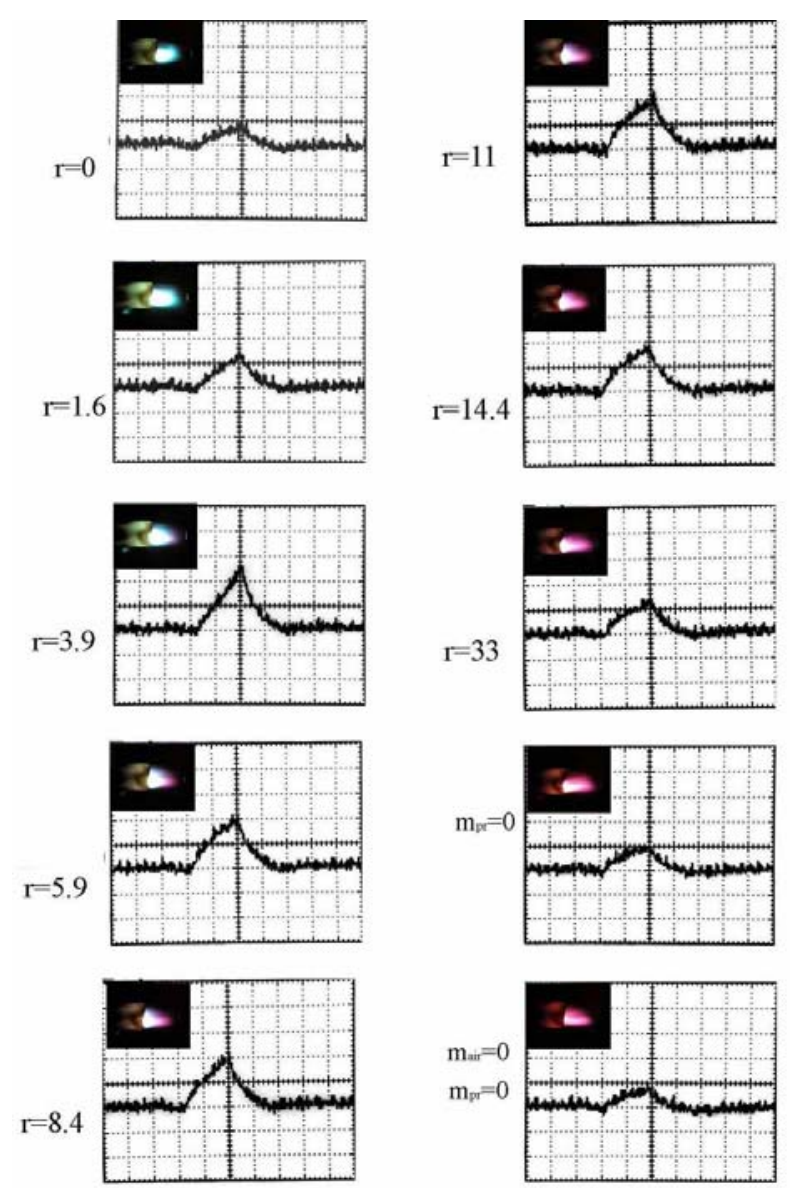

Figure 10. Waveforms $T_{\text {stag }}$ and appearance $M W$ discharge at combustion propane-air mixture over a range of air-fuel ratios, $r$.

\section{Experiments with air delivery to an internal mixing volume from the supersonic stream}

In this series of experiments, the air that is mixed with the propane was obtained from the freesteam through a hole of diameter $2 e$ in the nose of the vibrator, as can be seen in Fig.11.

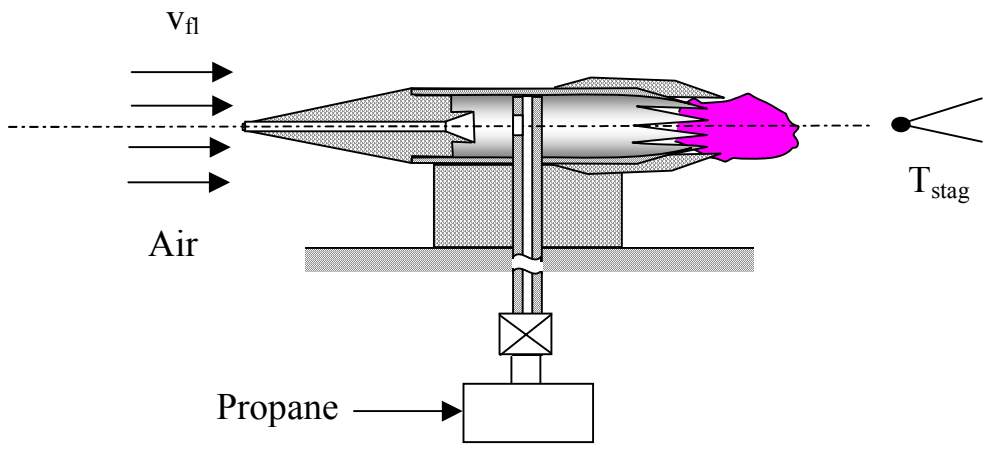

Figure 11. Scheme of the initiator's interior with injection of pure propane and air delivery from SS stream through inlet.

The air mass flow rate $\boldsymbol{m}_{\text {air }}$ was estimated based on comparisons of the optimum results of the previous experiments, and the size of the hole resulting in similar performance was estimated. First, an experiment was executed with air delivery to the vibrator channel for mixing with pure propane injected with a hole of $2 \boldsymbol{e}=1 \mathrm{~mm}$. For varying $\boldsymbol{m}_{p r}$, these results practically overlay with the results of the previous experiments. For example, in 
Fig.12 one can see (from left to right) typical waveforms and the appearance of the energy release region for several modes: in the absence of propane injection, at $\boldsymbol{m}_{\boldsymbol{p}}$ close to optimum value, and at an $\boldsymbol{m}_{\boldsymbol{p} r}$ value greater than the optimum one.
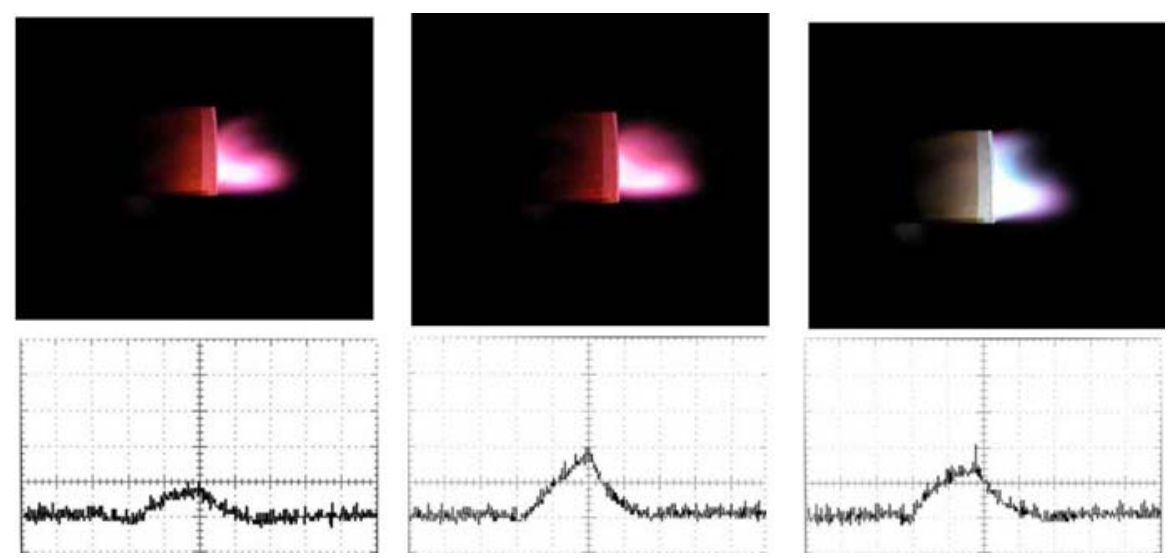

Figure 12. Typical waveforms and energy release region appearance for several modes (from left to right): in the absence of propane injection, at $m_{p r}$ close to optimum value, and at an $m_{p r}$ value greater than the optimum one.

The size $2 \boldsymbol{e}$ was increased to $1.5 \mathrm{~mm}$ in next series of experiments. For this configuration, the dependence of experimental results on $\boldsymbol{r}$ naturally shifted to a substantially higher optimum value for $\boldsymbol{m}_{\boldsymbol{p} r}$. In Fig.13 one can see a typical photograph of the energy release region of these experiments (left) and $\boldsymbol{T}_{\text {stag }}$ waveforms (right) for a case with only the discharge (the lower waveform) and for a case with the injection of the flammable mixture (upper waveform). It follows from the waveforms that the signal amplitude in the latter case substantially increased with respect to its value seen in Fig. 12 with $2 e=1 \mathrm{~mm}$.
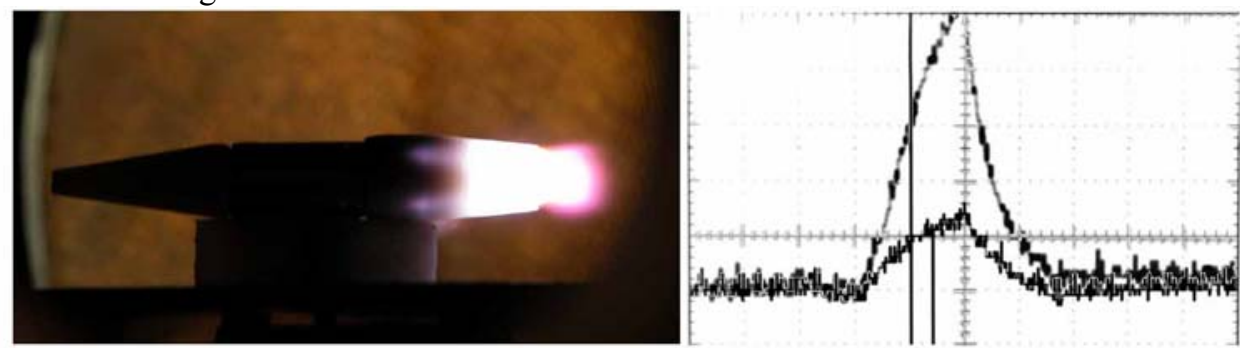

Figure 13. Typical photo of energy release region (to the left) and $T_{\text {stag }}$ waveforms (right) for only the discharge (lower waveform) and with injection of flammable mixture (upper waveform).

Several controlled experiments were executed during this experimental series. The propane mass flow rate $\boldsymbol{m}_{p r}$ was estimated with respect to the initial pressure of the propane in the injection section. We estimated the aerodynamic parameters of the air stream in the near wake of the vibrator along its axis by measuring $\boldsymbol{p}_{\text {stag }}$ at several locations in the wake. Measurements of $\boldsymbol{p}_{\text {stag }}$ in the channel allow one to estimate the mass flow rate of the air captured by the inlet device of the vibrator, which proved to be $\mathrm{m}_{\text {air }} \approx 3 \cdot 10^{-1} \mathrm{~g} / \mathrm{s}$. The optimum propane mass flow rate was determined using the maximum value of the measured $\boldsymbol{T}_{\text {stag }}$ at an axial point of energy release within the wake. The optimum value corresponded to $\mathrm{m}_{\mathrm{pr}} \approx 3 \cdot 10^{-2} \mathrm{~g} / \mathrm{s}$, so $\boldsymbol{r} \approx 10$.

In the series of experiments using this configuration, we obtained $\boldsymbol{p}_{\text {stag }}$ and $\boldsymbol{T}_{\text {stag }}$ at the optimum $\boldsymbol{m}_{\boldsymbol{p}}$ in a plane perpendicular to the stream located at a distance of $x=14.5 \mathrm{~mm}$ from the base of the dielectric nozzle. The waveforms are presented below with the pictures shown for a square grid $\boldsymbol{y}-\boldsymbol{z}$ with a step size of $2 \mathrm{~mm}$. In these results, the $\boldsymbol{y}$ axis is vertical and $\boldsymbol{z}$ axis is horizontal. The origin of the coordinate system in on the axis of the vibrator. The horizontal time scale is $0.2 \mathrm{~s} /$ div for all waveforms.

In Fig. 14 one can see the matrix of $\boldsymbol{p}_{\text {stag }}$ measurements for the case with burning of the discharge only; and in Fig. 15 one can the results for the case with the discharge and injection of the flammable mixture. The vertical scale in the waveforms is $10 \mathrm{mV} /$ div with a measurement sensitivity of $7.1 \mathrm{Torr} / \mathrm{mV}$. The fourth row from the bottom in this set corresponds to $y=0$, and the fifth column from the left corresponds to $z=0$. 


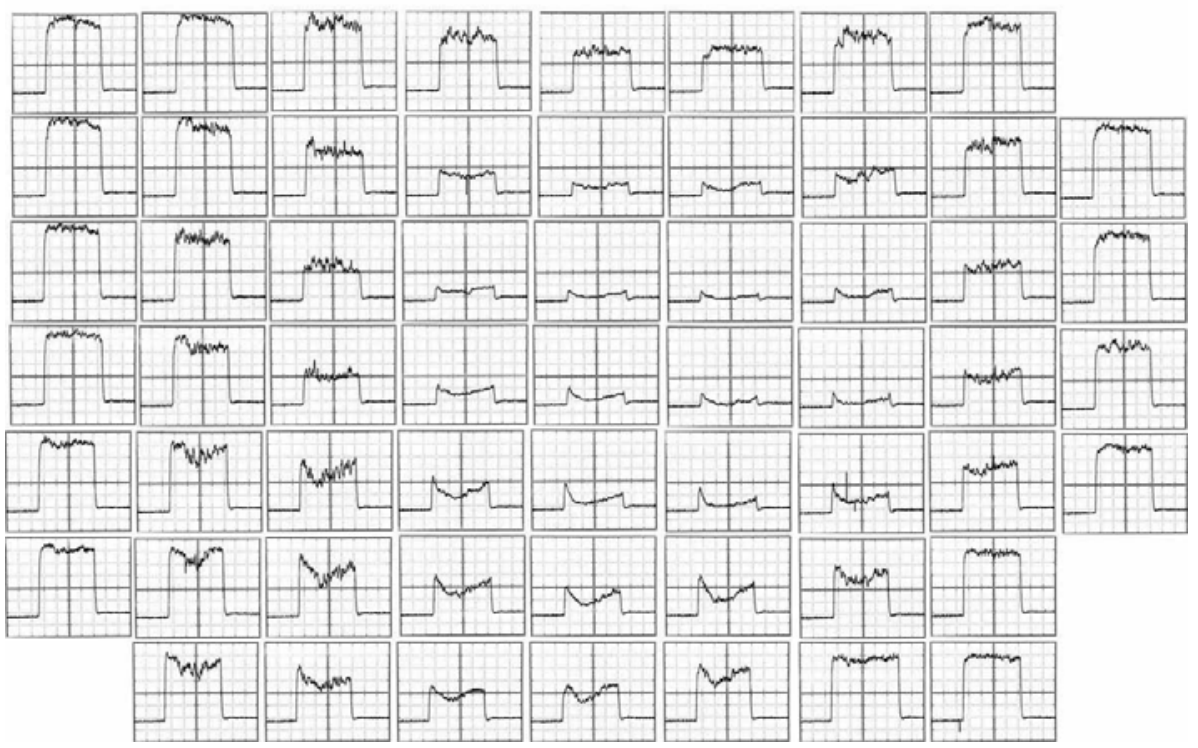

Figure 14. A matrix of $p_{\text {stag }}$ measurements for the case with only the discharge.

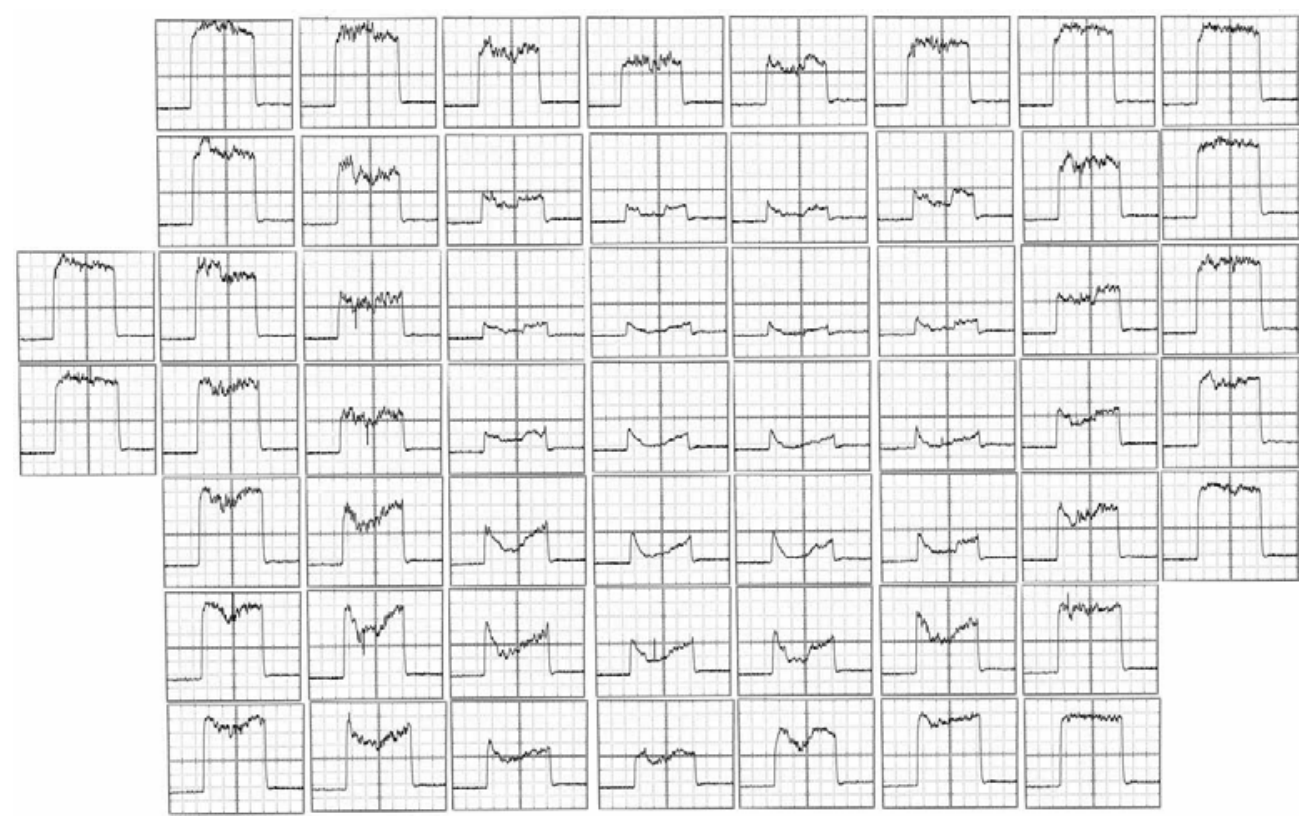

Figure 15. The matrix of $p_{\text {stag }}$ waveforms for the case with both the discharge and injection of the flammable mixture.

Fig. 16 and Fig. 17 present the results for two measurements simultaneously: $\boldsymbol{T}_{\text {stag }}$ using a thermocouple (upper curve) and $\boldsymbol{p}_{\boldsymbol{c}}$ (lower curve). The vertical scale in the waveforms is $10 \mathrm{mV} / \mathrm{div}$ for $\boldsymbol{p}_{\boldsymbol{c}}$ at $S=7.1 \mathrm{Torr} / \mathrm{mV}$ and at $2 \mathrm{mV} / \mathrm{div}$ for the thermocouple measurement. The initial level of $\boldsymbol{p}_{\boldsymbol{c}}$ in the waveforms corresponds to $\boldsymbol{p}_{\mathrm{c}}=114$ Torr. We used a value for the chromel-alumel thermocouple sensitivity of $\alpha=24.5 \mathrm{~K} / \mathrm{mV}$ together with the temporal variation of the waveforms to transform the raw data from the thermocouple to values of $\boldsymbol{T}_{\text {stag }}$ [2]. 


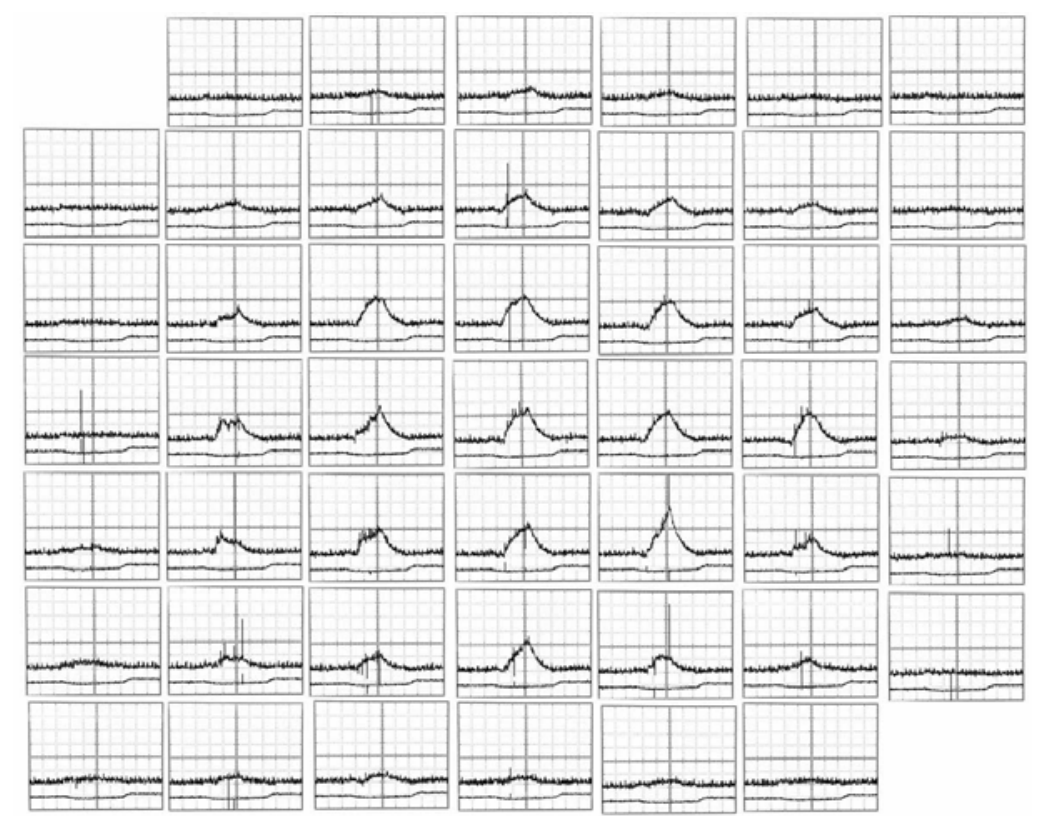

Figure 16. The matrix of $T_{\text {stag }}$ (the upper one) and $p_{c}$ waveforms for the case with only the discharge.

Fig. 16 corresponds to the case with only the MW discharge. The fourth row from the bottom corresponds to $y=0$, and the fourth column from the left corresponds to $z=0$. The maximum increase in temperature along the axis $y=z=0$ is estimated to be $\Delta T_{\text {dis } \max }=270 \mathrm{~K}$. Fig. 17 presents the results for the case with injection of the flammable mixture into the discharge region. The fifth row from the bottom corresponds to $y=0$, and the fourth column from the left corresponds to $z=0$. The maximum increase in temperature along the axis at $y=z=0$ in this case is estimated to be $\Delta T_{\max }=910 \mathrm{~K}$. So, combusting the propane results in an additional temperature increase of $\Delta T_{\text {com max }}$ $=640 \mathrm{~K}$ at this location.

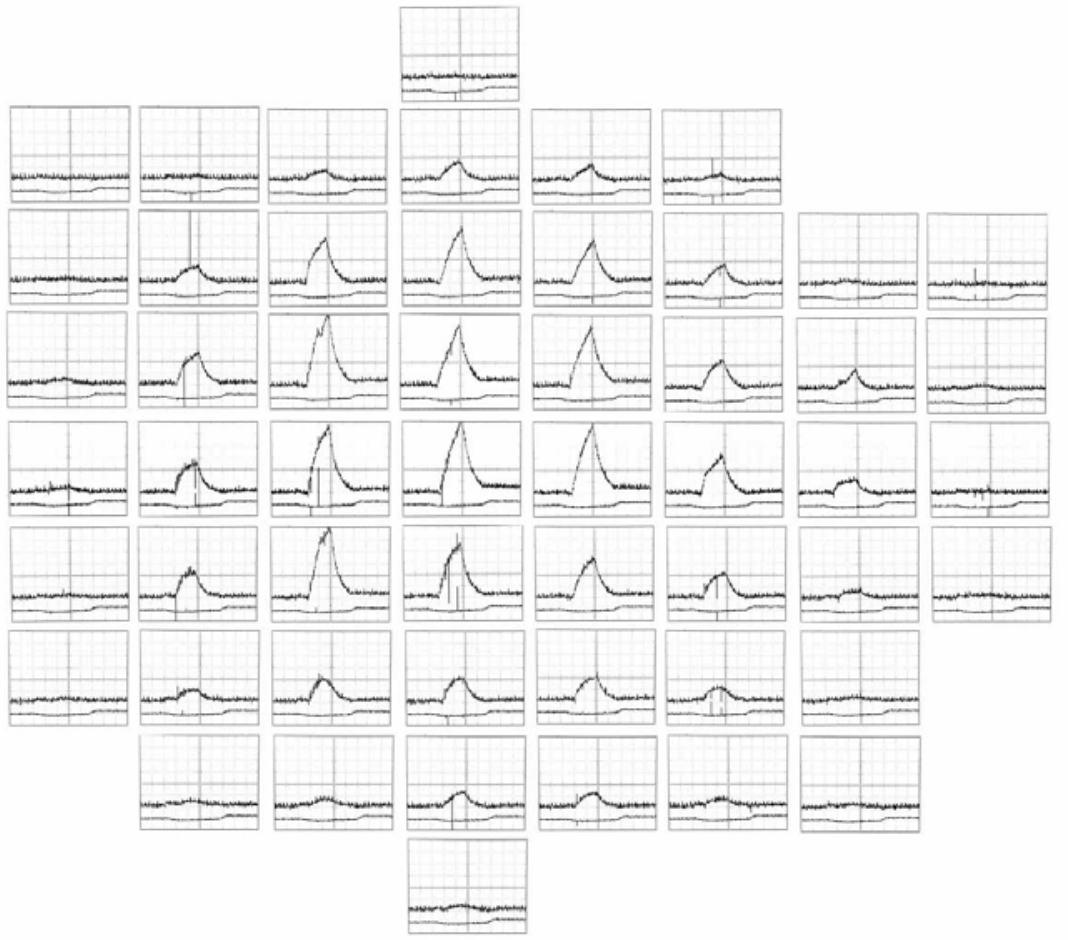

Figure 17. The matrix of $T_{\text {stag }}$ (upper curves) and $p_{c}$ waveforms (lower curves) for case with the discharge and injection of the flammable mixture. 
In Fig. 18 one can see the relative distributions of $\boldsymbol{p}_{\text {stag }}$ and $\boldsymbol{T}_{\text {stag }}$ processed with the help of the data from Fig. 16 and Fig. 17 for the case with the discharge only (left) and the case with the discharge and injection of the flammable mixture. These results were obtained in experiments conducted at $p_{1}=1 \mathrm{~atm}$ and $T_{1}=300 \mathrm{~K}$. This experimental data allows one to calculate the distributions of $\boldsymbol{T}_{s t}, \boldsymbol{M}, \mathbf{v}$ and the density of molecules $\boldsymbol{n}$ in the plane of the measurements. In turn, this data allows one to estimate the degree to which the incident EM field power is transformed to gas heating in the discharge $\boldsymbol{P}_{\text {dis }}$ and the degree of propane combustion power $\boldsymbol{P}_{\text {com }}$. The estimated values are $P_{\mathrm{dis}} \approx 210 \mathrm{~W}$ and $P_{\text {com }} \approx 800 \mathrm{~W}$ for this case. From these values, the efficiencies of EM field energy use and propane combustion are $14 \%$ and $57 \%$ at $P_{\mathrm{MW}}=1.5 \mathrm{~kW}$ and $P_{\text {com max }}=1.4 \mathrm{~kW}$, which corresponds to complete propane combustion.

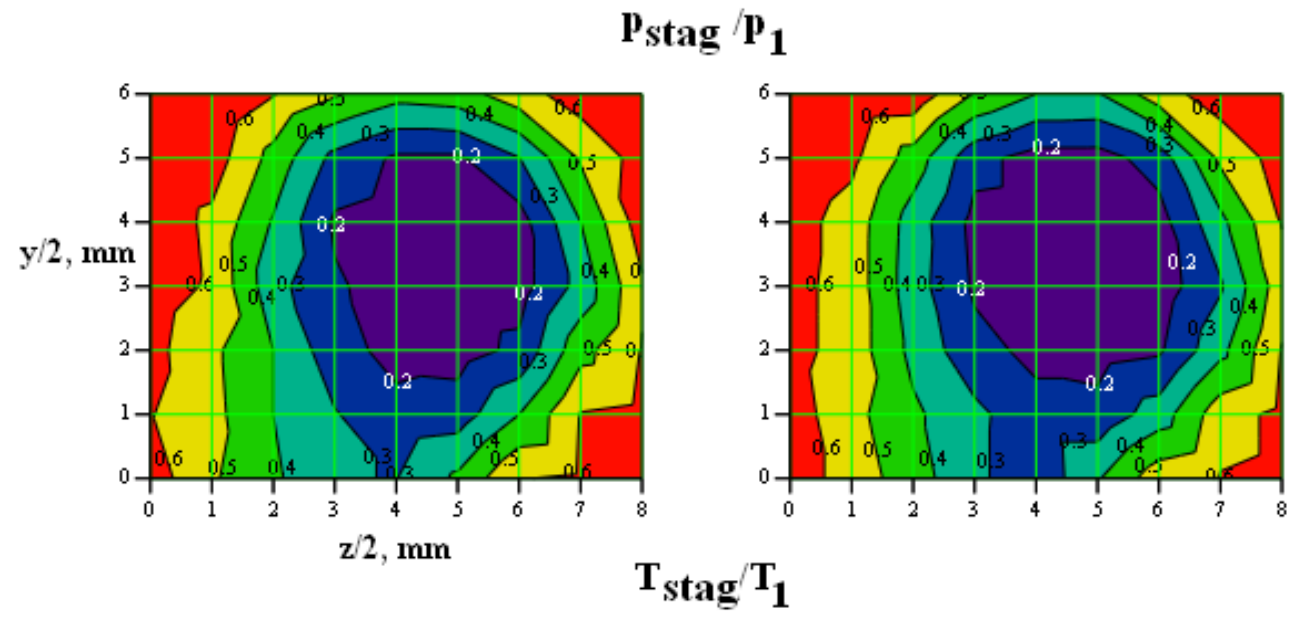

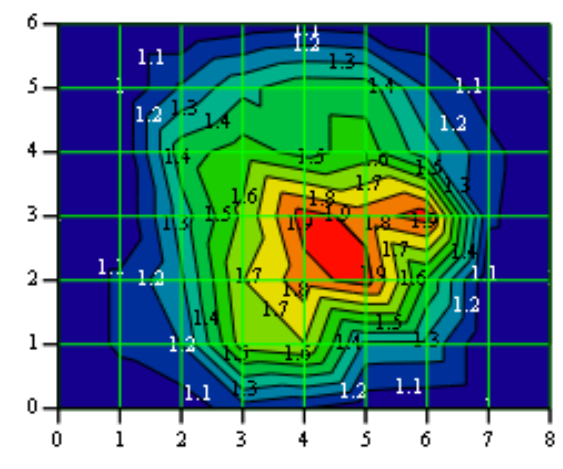

a)

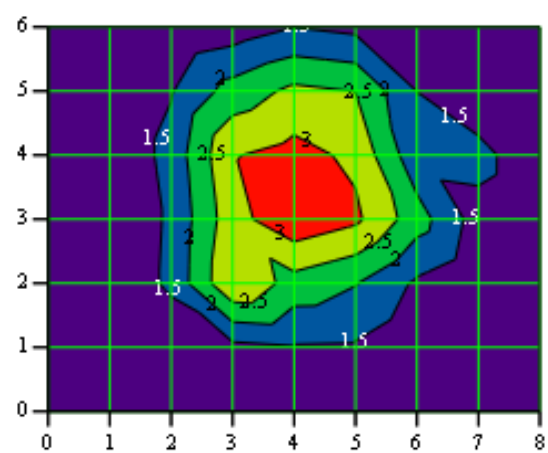

b)

Figure 18. Stagnation pressure and stagnation temperature in a transverse cross-section at a distance of. $4 \mathrm{~cm}$ from the injection base: a) air is injected through the mixer to the discharge area; b) propane-air mixture is injected to the discharge area.

\section{Characteristics of a torch in the supersonic stream}

The data from the stagnation pressure and temperature measurements allow one to determine the distributions of the main parameters in the stream cross-section, namely, the velocity, static temperature, density, Mach number and input energy power. The distributions obtained are presented in Fig.19 with the temperature and density normalized to values for the standard atmosphere $\boldsymbol{T}_{\boldsymbol{1}}$ and $\boldsymbol{n}_{1}$, and velocity normalized by $V 1$ equal to the sonic velocity at standard conditions divided by $\sqrt{\gamma}$.

One can see from the distributions that the static temperature of the torch is $1000 \mathrm{~K}$ on the axis in the measurement cross section, which is lower than the mixture combustion temperature. The stream velocity on the stream axis equals approximately $200 \mathrm{~m} / \mathrm{s}$ compared to an external flow velocity of $490 \mathrm{~m} / \mathrm{s}$. This velocity difference demonstrates the intense interaction that takes place between the torch and the external supersonic flow, which leads to a decrease in the average torch temperature and the delivery of additional air to the combustion zone. Apparently, this phenomenon explains the fact that the maximum combustion power is observed with the injection 
of a mixture with a propane content exceeding the stoichiometric value by a factor of two. The additional air coming from the external flow results in an increase in the completeness of the propane combustion in the torch.
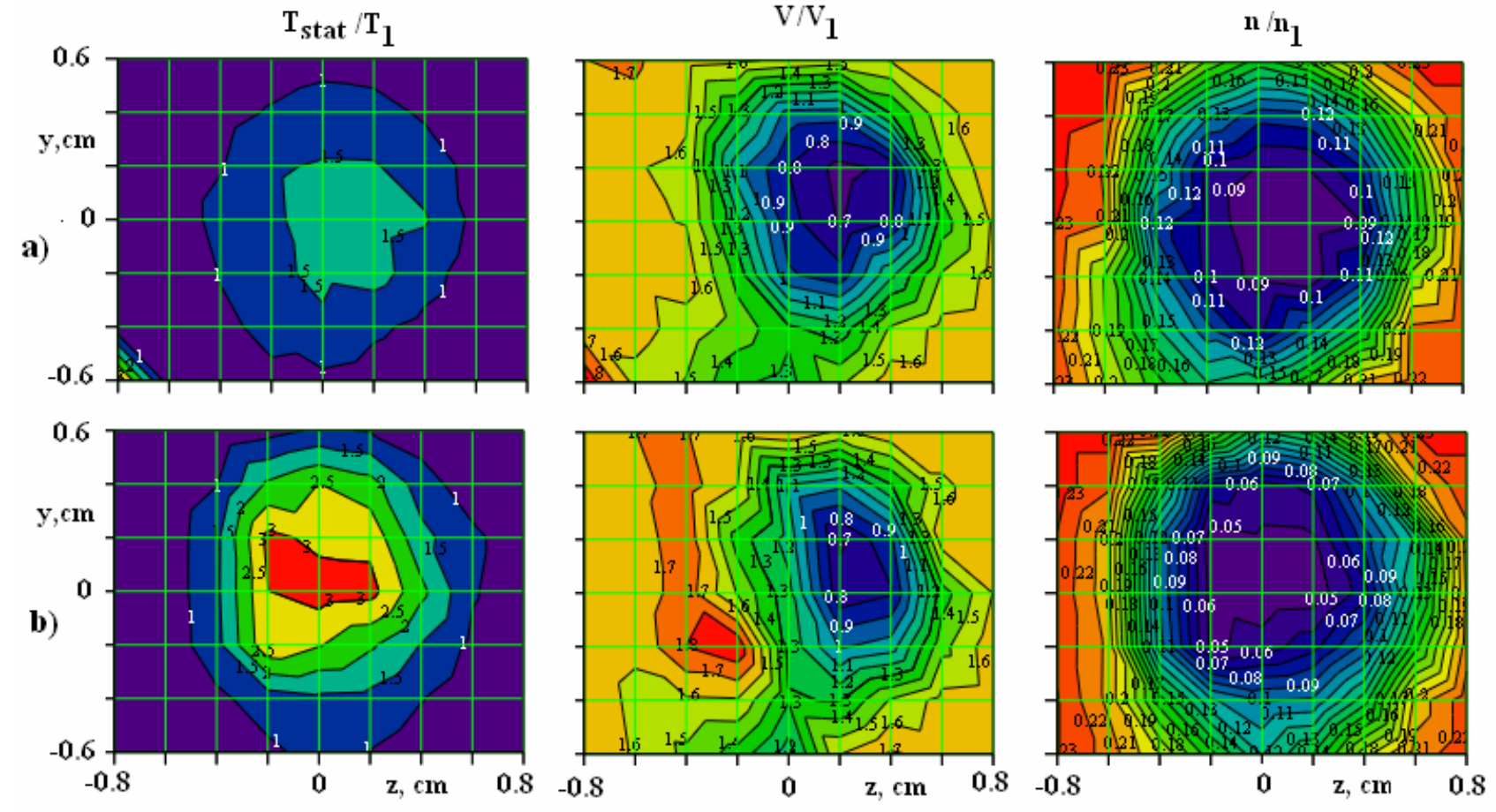

Figure 19. Distribution of stream parameters (static pressure, velocity and density) in transverse cross section at $x=1.2 \mathrm{~cm}$. a) without propane injection, b) with propane injection.

The power from the discharge and combustion is determined by the integral over the cross section

$W=\iint n(y, z) \cdot V(y, z) \cdot C_{p} \cdot\left(T_{\text {stag }}-1\right) \cdot d y \cdot d z$

The difference in the integral values for experiments with and without propane delivery allows one to determine the value of the combustion power. The values that were obtained confirm the estimates made above.

It should be noted the key role of the insulator covering the base of the metal vibrator. Its presence creates conditions for the homogeneous burning of the MW discharge inside this insulator tube. The temperature of the gas mixture inside the mouthpiece is high not only in separate streamers but in the full cross-section of the outlet. The high value of the mixture temperature is the main reason for the rapid combustion in the high speed flow. In addition to the high gas temperature in the MW discharge, it is believed that some role is played by the ultraviolet radiation generated in the discharge.

The appearance of a homogeneous discharge inside the dielectric cover can be explained by the significant changes that result in the gas dynamic picture of the flow near the base of the vibrator. The many apexes in the aft end of the vibrator needed for initiation of the discharge result in the appearance of local separation zones inside the vibrator channel. The circumferential and longitudinal variations of the channel radius created by these apexes perturb the flow inside the cover resulting is turbulence. This phenomenon leads to the creation of a more uniform MW discharge in the circumferential direction but also causes more favorable conditions for the mixing and combustion of the flammable mixture. Of course the details associated with this complex interaction require additional investigation.

\section{Conclusions}

The results of the experimental program have conclusively demonstrated that use of a deeply undercritical microwave discharge initiated by a vibrator located in the supersonic flow can be used to effectively increase the combustion of an air/propane mixture in cold supersonic flow. A promising configuration was demonstrated wherein air is delivered to an internal mixture region inside the vibrator from the external supersonic stream through an 
intake in the nose of the vibrator. The degree of combustion substantially increases with the use of a dielectric "combustion chamber" in the base area of the vibrator.

The undertaken investigations of propane-air mixture combustion in a microwave discharge allowed demonstration of the stable operation of a hot torch of combustion products in a supersonic stream in steady-state operation. The combustion of the mixture in the discharge and within the torch takes place at a mixture velocity from 100 to $200 \mathrm{~m} / \mathrm{s}$ for cold airflows.

The realization of quasi-stationary combustion at a delivered mixture velocity of $100 \mathrm{~m} / \mathrm{s}$ allows one to postulate the influence of hard optical radiation on the process of combustion of the propane-air mixture in the area of the microwave discharge plasma. This influence can substantially increase the velocity range for the mixture combustion and significantly widen the concentration limits for ignition.

Further investigations of the ignition characteristics in a microwave stationary discharge are needed to determine: the velocity of the combustion front in a supersonic mixture stream in the zone of microwave radiation; the degree of propane combustion completeness with respect to mixture and discharge parameters; and the characteristics at higher initial temperatures and velocities.

\section{Acknowledgments}

This work was supported by European Office of Research and Development (EOARD), Project \#2429p

\section{References}

\footnotetext{
${ }^{1}$ Igor Esakov, Lev Grachev, Kirill Khodataev and D.M.Van Wie, "Experiments on propane ignition in high-speed airflow using a deeply undercritical microwave discharge", 42 ${ }^{\text {nd }}$ AIAA Aerospace Sciences Meeting and Exhibit, 5-8 January 2004, Reno, Nevada, AIAA 2004-840.

${ }_{2}^{2}$ Igor Esakov, Lev Grachev, Kirill Khodataev and D.M.Van Wie, "Efficiency of microwave discharges for propane ignition in cold high-speed airflow", 43 ${ }^{\text {nd }}$ AIAA Aerospace Sciences Meeting and Exhibit, 10-13 January 2005, Reno, Nevada, AIAA 2005-989.
} 